\title{
Harmine combined with paclitaxel inhibits tumor proliferation and induces apoptosis through down-regulation of cyclooxygenase- 2 expression in gastric cancer
}

\author{
XIAO-JUAN YU ${ }^{1 *}, \mathrm{KUN} \mathrm{SUN}^{2 *}$, XIAO-HE TANG ${ }^{2}, \mathrm{CUN}$-JIN ZHOU ${ }^{2}, \mathrm{HUI} \mathrm{SUN}^{2}$, \\ ZHE YAN $^{2}$, LING FANG ${ }^{2}$, HONG-WEN WU ${ }^{2}$, YI-KUI XIE ${ }^{2}$ and BIN GU ${ }^{1}$ \\ ${ }^{1}$ Department of Emergency, People's Hospital of Taizhou, Jiangsu 225300; \\ ${ }^{2}$ Department of Gastroenterology, First Hospital of Zibo, Shandong 255200, P.R. China
}

Received February 26, 2015; Accepted March 10, 2016

DOI: $10.3892 / \mathrm{ol} .2016 .4696$

\begin{abstract}
Cyclooxygenase-2 (COX-2) serves an important role in the carcinogenesis and progression of gastric cancer. Harmine (HM) and paclitaxel (PTX) are reported as promising drug candidates for cancer therapy, but whether a synergistic anti-tumor effect of HM combined with PTX exists in human gastric cancer remains unknown. The present study evaluated the effects of HM and/or PTX on cell proliferation and apoptosis in a gastric cancer cell line, SGC-7901. HM and PTX inhibited cell proliferation in a dose-dependent manner. Both HM and PTX alone induced apoptosis in gastric cancer cells. The combination of HM and PTX exerted synergistic effects on proliferation inhibition and apoptosis induction in SGC-7901 cells, with down-regulation of COX-2, PCNA and Bcl-2 and up-regulation of Bax expression. The results indicated that combination chemotherapy using HM with PTX exerts an anti-tumor effect for treating gastric cancer. The combination of the two drugs inhibits gastric cancer development more effectively than each drug alone through down-regulation of COX-2 expression.
\end{abstract}

\section{Introduction}

Gastric cancer has been a significant health problem worldwide due to its poor prognosis and increasing incidence (1). According to the latest literature, 800,000 cancer-associated mortalities are caused by gastric cancer each year globally,

Correspondence to: Professor Yi-Kui Xie, Department of Gastroenterology, First Hospital of Zibo, 4 Emeishan East Road, Zibo, Shandong 255200, P.R. China

E-mail: xyk6351@163.com

Professor Bin Gu, Department of Emergency, People's Hospital of Taizhou, 399 Hailing Road, Taizhou, Jiangsu 225300, P.R. China

E-mail: zaijian930@sina.com

*Contributed equally

Key words: harmine, paclitaxel, cyclooxygenase-2, gastric cancer, apoptosis making it the second leading cause of cancer-associated mortalities in the world $(2,3)$. At present, chemotherapy has become one of the major means for treating gastric cancer of middle and advanced stages (4,5). As a standard anticancer drug, paclitaxel (PTX) serves a significant role in the treatment of a number of tumors. As reported in latest studies, the efficiency of single anticancer drug-paclitaxel reaches $11-23 \%$ in treating the gastric cancer of middle and advanced stages, while that of drug combination therapy is 50-60\% (6). Harmine (HM), originally isolated from the seeds of Peganum harmala, is a tricyclic compound belonging to the $\beta$-carboline alkaloids. It inhibits the proliferation of tumor cells and induces apoptosis, and it performs well in reducing angiogenesis, tumor promotion and mutation $(7,8)$. It has become a new focus in the chemoprevention study about cancer in recent years. Recent studies have demonstrated that HM possessed significant anti-tumor potential both in vitro and in vivo $(9,10)$. However, the synergistic anti-tumor effect of a combination of HM and PTX on human gastric cancer remains unknown.

Cyclooxygenase (COX), a key enzyme in the conversion of arachidonic acid to prostaglandins (PGs) and other eicosanoids, exists as two isoforms: Constitutive COX-1 and mitogen-inducible COX-2. COX-2 is also constitutively expressed in gastric cancer and is related to cell proliferation and apoptosis, tumor invasiveness and metastasis (11). Our previous studies have demonstrated that COX-2 inhibition by selective COX-2 inhibitors or small interfering RNA (siRNA) could suppress cell proliferation and induces apoptosis in human gastric cancer cells $(12,13)$. Recently, we demonstrated that HM induced apoptosis of gastric cancer cells by down-regulating COX-2 expression (14).

In the present study, a human gastric cancer cell line SGC-7901, in which COX-2 was expressed (15), was selected to examine whether PTX in combination with HM exerts synergistic anti-tumor effects on human gastric cancer cells in vitro and in vivo and to further explore the probable mechanism.

\section{Materials and methods}

Reagents. HM, PTX (purity >98\%), dimethylsulfoxide (DMSO), and 3-(4,5-dimethylthiazol-2-yl)-2,5-diphenyl tetrazolium 
bromide (MTT) were purchased from Sigma-Aldrich (St. Louis, MO, USA). The chemical structures of HM and PTX are shown in Fig. 1. Stock solutions of HM were produced in DMSO at a final concentration of DMSO $(\leq 0.1 \%)$ and sterilized by passage through a $0.22 \mu \mathrm{m}$ pore size filter (Immobilon; EMD Millipore, Bedford, MA, USA), diluted with culture media before use. RPMI-1640 medium, fetal bovine serum (FBS) and penicillin/streptomycin were purchased from Gibco BRL (Grand Island, NY, USA). All other chemicals were of analytical grade and used without further purification.

Cell lines and culture. Human moderately differentiated SGC-7901 gastric cancer cell line was obtained from Shanghai Institute of Cell Biology (Shanghai, China). The cells were cultured in RPMI-1640 medium supplemented with 10\% FBS, 100 units $/ \mathrm{ml}$ penicillin $\mathrm{G}$ and $100 \mu \mathrm{g} / \mathrm{ml}$ streptomycin at $37^{\circ} \mathrm{C}$ in a humidified incubator with $5 \% \mathrm{CO}_{2}$.

MTT assay. SGC-7901 cells (200 $\mu 1$ of cell suspension per well) were seeded at a density of $5 \times 10^{3}$ cells/well in 96-well plates and incubated overnight in 10\% FBS medium. The cells were then treated with different concentrations of $\mathrm{HM}$ or PTX in serum-free conditions. Untreated cells in serum-free medium were used as controls. After incubation for $48 \mathrm{~h}$ at $37^{\circ} \mathrm{C}$, the cell proliferation was determined by the MTT assay as described in our previous study (15).

DAPI staining. Equal numbers of SGC-7901 cells $\left(1 \times 10^{6}\right.$ cells/well $)$ were plated in 6-well plates and then incubated with $2 \mathrm{ng} / \mathrm{ml} \mathrm{PTX}, 4 \mu \mathrm{g} / \mathrm{ml} \mathrm{HM}$, or a combination of these two drugs for $48 \mathrm{~h}$ and then washed once in phosphate buffer saline (PBS) followed by fixation in cold methanol: acetone (1:1) for $5 \mathrm{~min}$. After washing twice in PBS for $5 \mathrm{~min}$, these cells were stained with $4 \mu \mathrm{g} / \mathrm{ml}$ DAPI for $10 \mathrm{~min}$ at room temperature and subsequently examined by fluorescence microscopy (Eclipse E-800; Nikon, Tokyo, Japan). Apoptotic cells were identified by chromatin condensation and nuclear fragmentation.

Flow cytometry analysis. Equal numbers of SGC-7901 cells $\left(1 \times 10^{6}\right.$ cells/well) were plated in 6-well dishes and then incubated with $2 \mathrm{ng} / \mathrm{ml}$ PTX and/or $4 \mu \mathrm{g} / \mathrm{ml} \mathrm{HM}$ or a combination of these two drugs for $48 \mathrm{~h}$. The cells were washed with PBS, stained with annexinV-FITC and propidium iodide (PI) using the AnnexinV-FITC kit (Bender Medsystem, Vienna, Austria). The cells were then subjected to flow cytometry according to the manufacturer's instructions and the stained cells were analyzed by FACScan flow cytometer (BD Biosciences, San Diego, CA, USA).

Western blot analysis. Proteins were extracted from cells or tumors and western blot analyses were performed as described in our previous reports (15). Cells $\left(1 \times 10^{6}\right.$ cells/well) were treated with $2 \mathrm{ng} / \mathrm{ml} \mathrm{PTX}, 4 \mu \mathrm{g} / \mathrm{ml} \mathrm{HM}$ or a combination of these two drugs for $48 \mathrm{~h}$ at $37^{\circ} \mathrm{C}$ in a humidified incubator with an atmosphere of $5 \% \mathrm{CO}_{2}$. Cells were then washed twice with ice cold PBS and protein extraction was performed by lysis in RIPA buffer [150 mM NaCl, $1 \%$ (v/v) NP40, 0.5\% (w/v) sodium deoxycholate, $0.1 \%(\mathrm{w} / \mathrm{v})$ sodium dodecyl sulfate (SDS), $50 \mathrm{mM}$ Tris $\mathrm{HCl}(\mathrm{pH} 8), 10 \mathrm{mM}$ EDTA and $1 \mathrm{mM}$ phenyl methylsulfonyl fluoride; Sigma-Aldrich] for $30 \mathrm{~min}$ at $4^{\circ} \mathrm{C}$, followed by<smiles>COc1ccc2c(c1)[nH]c1c(C)nccc12</smiles>

B

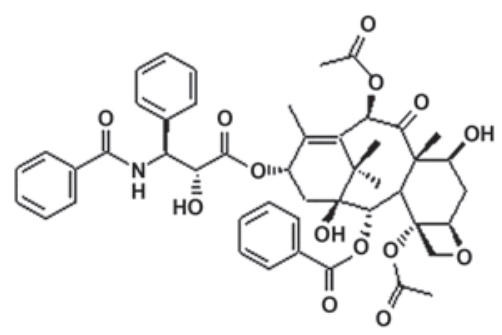

Figure 1. Chemical structure of (A) harmine and (B) paclitaxel.

centrifugation for $15 \mathrm{~min}$ at $12,000 \mathrm{x}$ g. Protein concentrations were determined using a bicinchoninic acid assay (Pierce Biotechnology, Inc., Rockford, IL, USA), according to the manufacturer's instructions. Subsequently, proteins $(60 \mu \mathrm{g})$ were loaded onto a $10 \%$ (w/v) SDS polyacrylamide gel, electrophoresed and transferred onto a polyvinylidene fluoride membranes (EMD Millipore) which was then blocked for $2 \mathrm{~h}$ at room temperature with blocking buffer [Tris-buffered saline containing 0.1\% (v/v) Tween-20 (Sigma-Aldrich) and 5\% (w/v) milk powder]. The following primary antibodies were applied at a dilution of 1:1,000 for $1 \mathrm{~h}$ at room temperature or overnight at $4^{\circ} \mathrm{C}$ : Polyclonal rabbit anti-mouse COX-2 (cat no. 13314; Cell Signaling Technology, Inc., Beverly, MA, USA), PCNA (cat no. sc53407; Santa Cruz Biotechnology, Inc., Dallas, TX, USA), B-cell lymphoma-2 (Bcl-2; cat no. sc509; Santa Cruz Biotechnology, Inc.), Bcl2-associated X protein (Bax; cat no. sc20067; Santa Cruz Biotechnology, Inc.) and GAPDH (cat no. G5262; Sigma-Aldrich). Membranes were then incubated for $2 \mathrm{~h}$ with polyclonal goat anti-rabbit horseradish peroxidase-conjugated secondary antibodies (1:20,000 diltion; cat no. BA1000; Vector Laboratories, Inc., Burlingame, CA, USA) at $37^{\circ} \mathrm{C}$ in a humidified incubator with an atmosphere of $5 \% \mathrm{CO}_{2}$. Membranes were visualized using an enhanced chemiluminescence kit and signals were quantified by scanning densitometry (QuantityOne v4.6.2 software; Bio-Rad Laboratories, Hercules, CA, USA). The relative expression levels of COX-2, PCNA, Bax and Bcl-2 were normalized to that of GAPDH.

In vivo anti-tumor efficacy. Male athymic nude mice (6-8 weeks old and weighing 18-22 g) were purchased from Animal Center of Nanjing Medical University (Nanjing, China). The mice were bred under specific pathogen-free (SPF) conditions and all experimental procedures were performed in accordance with the Guide for the Care and Use of Laboratory Animals (NIH publication no. 80-23, revised 1996) and the institutional ethical guidelines for animal experiments. The mice were subcutaneously injected into the left axillary space with $0.1 \mathrm{ml}$ of cell suspension containing 4-6x10 ${ }^{6}$ SGC-7901 cells. Seven days after implantation of tumor cells (when the tumor size was $\sim 0.1-0.2 \mathrm{~cm}^{3}$ ), mice were randomly divided into four groups $(n=6)$ to receive different treatments. The experimental mice were injected intraperitoneally with PTX 

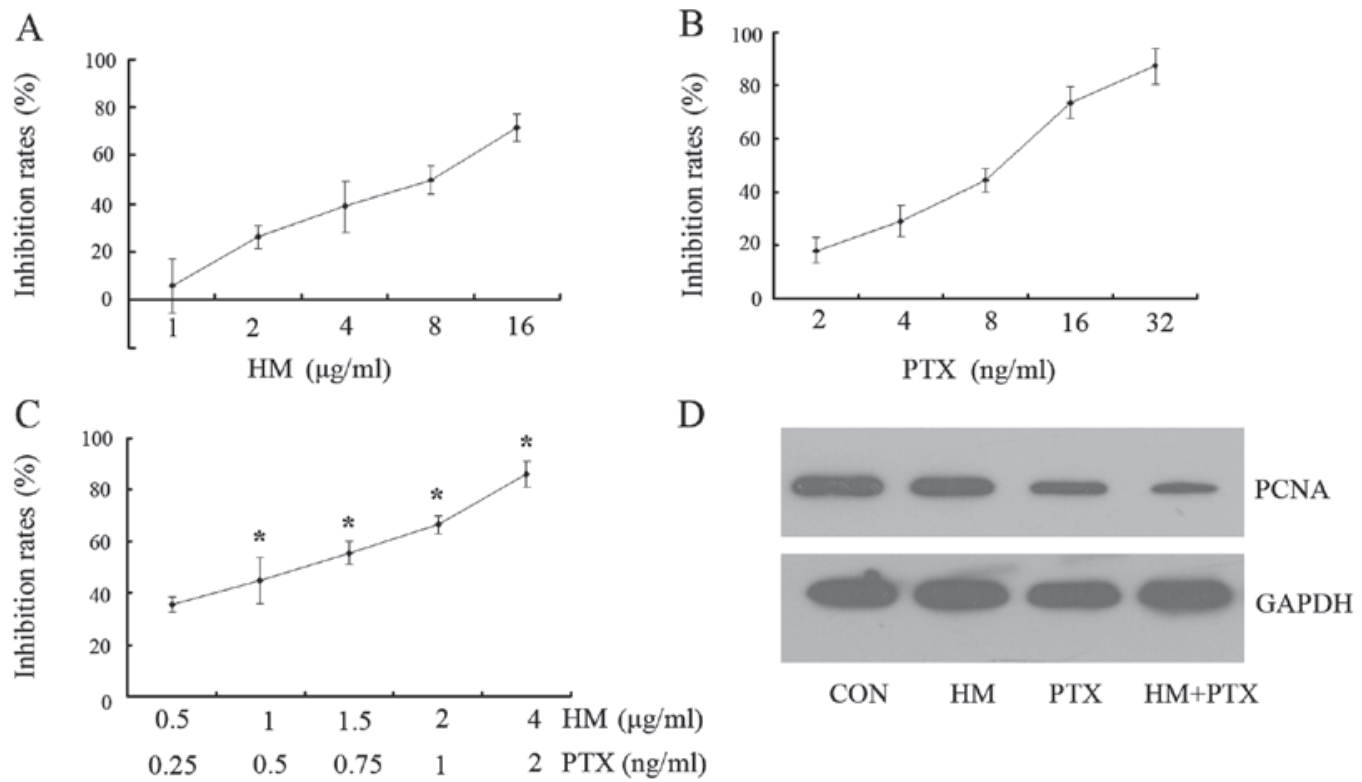

Figure 2. Effects of HM and PTX on cell proliferation. Dose-dependent effect of (A) HM and (B) PTX on cell proliferation. (C) Effect of the combination of these two drugs on cell proliferation. " $\mathrm{P}<0.05$ vs. PTX or HM. (D) Effects of HM and PTX on PCNA expression. HM, harmine; PTX, paclitaxel.

A

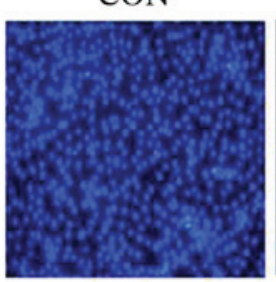

$\mathrm{B}$

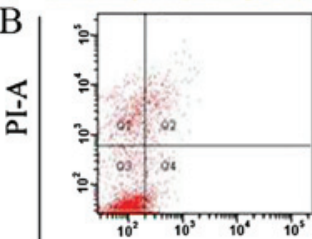

$\mathrm{HM}$
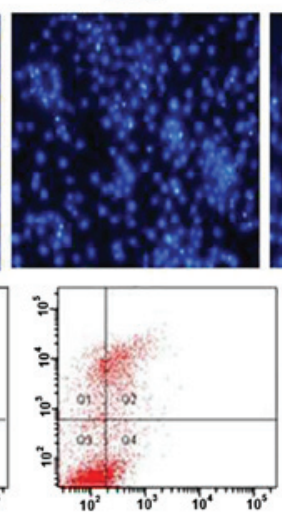

PTX
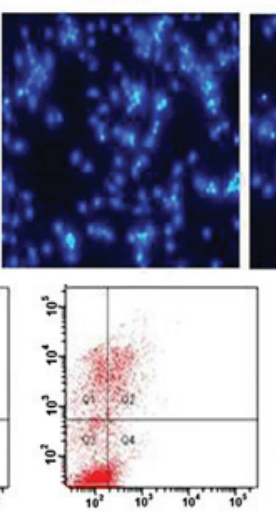

$\mathrm{C}$

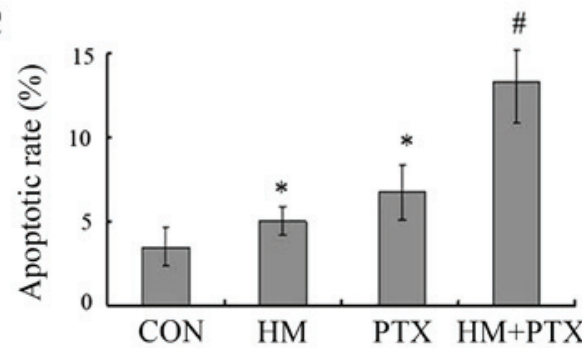

Figure 3. Effects of HM and PTX on the apoptosis of gastric cancer cells. Cells were treated with $2 \mathrm{ng} / \mathrm{ml} \mathrm{PTX} \mathrm{and/or} 4 \mu \mathrm{g} / \mathrm{ml} \mathrm{HM}$ for $48 \mathrm{~h}$. (A) Morphological changes in apoptotic cells were observed by DAPI staining and numbers were further measured by (B) flow cytometric analysis. (C) Histogram representing the mean \pm standard deviation of apoptosis rates obtained from 3 independent experiments. ${ }^{*} \mathrm{P}<0.05$, vs. control; ${ }^{*} \mathrm{P}<0.05$ vs. PTX or HM. HM, harmine; PTX, paclitaxel.

(10 mg/kg, daily, 3 days per week for 2 weeks), $\mathrm{HM}(30 \mathrm{mg} / \mathrm{kg}$, daily, 5 days per week for 2 weeks) and a combination of PTX $5 \mathrm{mg} / \mathrm{kg}$ and HM $20 \mathrm{mg} / \mathrm{kg}$, daily, 3 days per week for 2 weeks. Control mice were treated with an equal volume of normal saline. Tumors were measured with calipers at 3-day intervals and the volumes were calculated using the following formula: (the shortest diameter) ${ }^{2} \mathrm{x}$ (the longest diameter) $\mathrm{x} 0.5$.
Statistical analysis. Statistical analyses were performed using the SPSS software package (version 11; SPSS Inc., Chicago, IL, USA). The data are presented as mean \pm standard deviation (SD), and were analyzed using two-tailed Student's $t$-test or one-way analysis of variance (ANOVA) with Dennett's multiple comparison tests. $\mathrm{P}<0.05$ was considered to indicate a statistically significant difference. 

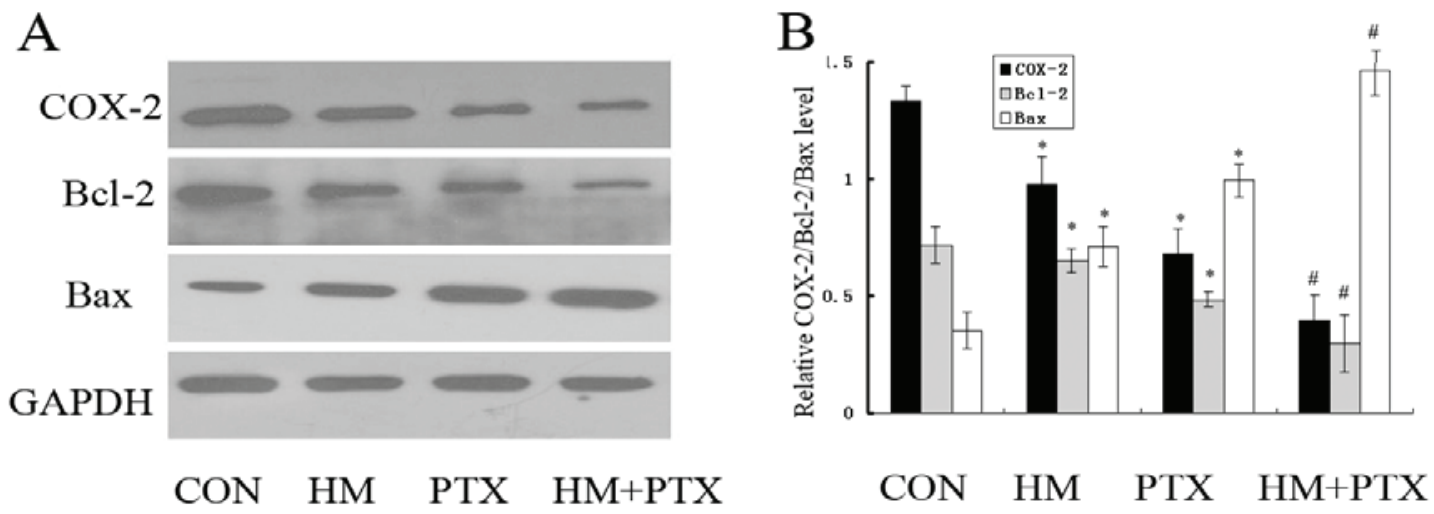

Figure 4. Effects of HM and PTX on COX-2, Bcl-2 and Bax protein expression levels in SGC-7901 cells in vitro. Cells were treated with $2 \mathrm{ng} / \mathrm{ml} \mathrm{PTX} \mathrm{and/or}$ $4 \mu \mathrm{g} / \mathrm{ml} \mathrm{HM}$ for $48 \mathrm{~h}$. HM, harmine; PTX, paclitaxel. (A) Western blot analysis and (B) quantification of relative COX-2, Bcl-2 and Bax expression. Data are presented as the mean \pm standard deviation from three independent experiments. ${ }^{*} \mathrm{P}<0.05$ vs. $\mathrm{CON}$; ${ }^{"} \mathrm{P}<0.05$ vs. PTX or HM.

\section{Results}

Effects of HM and PTX on the proliferation of SGC-7901 cells. MTT assay was used to analyze metabolic activity in proliferating cells. Either HM or PTX inhibited the cell proliferation in a dose-dependent manner (Fig. 2A and B). The inhibitory effects on the cell proliferation were significantly enhanced when SGC-7901 cells were treated with a combination of HM with PTX (Fig. 2C). As demonstrated in Fig. 2, the suppression rates of HM and PTX were 18.0 and $38.7 \%$, respectively. When HM and PTX were combined, the suppression rate reached $74.5 \%$, which was significantly higher than HM or PTX alone ( $\mathrm{P}<0.05 ;$ Fig. $2 \mathrm{C})$. Furthermore, HM combined with PTX reduced PCNA expression in gastric cancer cells (Fig. 2D).

Effects of HM and PTX on the apoptosis of SGC-7901 cells. Morphological changes in apoptotic cells were observed by DAPI staining. As shown in Fig. 3A, the nucleus of untreated control cells were large and round without condensation or fragmentation, whereas the nucleus from the HM or PTX treated cells were condensed and fragmented, emitted bright fluorescence, which is indicative of early apoptosis. In the combination group, changes in cellular morphology were much stronger than that of either drug applied individually.

To further confirm the apoptotic combined effects of HM and PTX, fluorescent Annexin V-FITC/PI double staining was performed. When cells undergo apoptosis, a phosphatidylserine residue normally on the inside of the plasma membrane flips to the outside and are specifically recognized by annexin V. Counterstaining by PI allows the discrimination of apoptotic from necrotic cells. Necrotic cells will be stained only with PI, whereas early apoptotic cells will be stained with annexin $\mathrm{V}$ and late apoptotic cells will be stained with both annexin V and PI. As demonstrated in Fig. 3B, the lower right panels correspond to apoptotic cells which have high FITC and low PI signals. Both HM and PTX induced apoptosis in SGC-7901 cells $(\mathrm{P}<0.05$ vs.
A

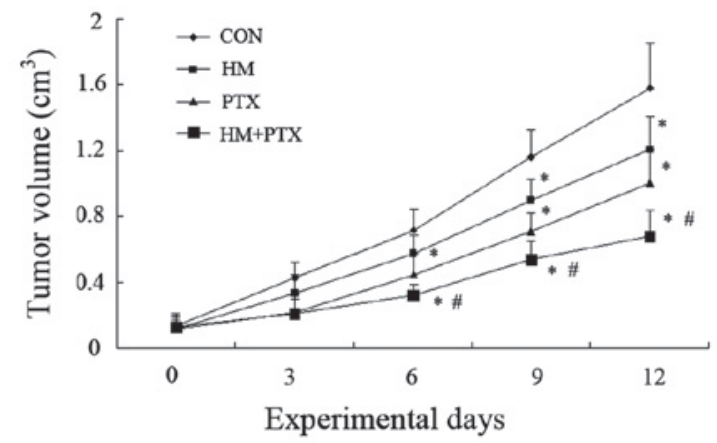

B

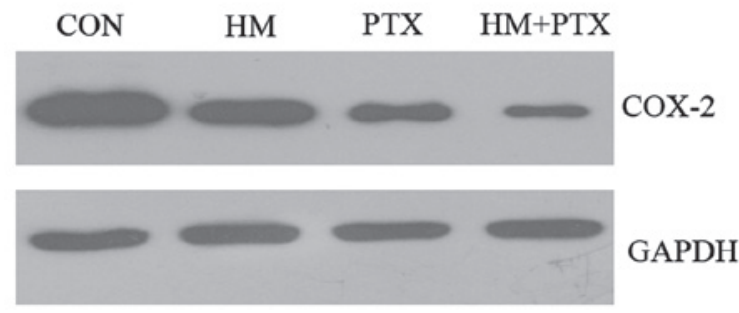

Figure 5. Effects of HM and PTX on tumor growth in vivo. (A) Tumor growth curve. (B) Western blot analysis demonstrated that both HM and PTX decreased COX-2 expression. Data are presented as the mean \pm standard deviation from 6 mice. ${ }^{*} \mathrm{P}<0.05$ vs. control (saline). ${ }^{\#} \mathrm{P}<0.05$ vs. PTX or HM. HM, harmine; PTX, paclitaxel.

control for HM and PTX), the combination of two drugs further enhanced the apoptosis ratio $(\mathrm{P}<0.05, \mathrm{HM}+\mathrm{PTX}$ vs. HM and PTX) (Fig. 3C).

Effects of HM and PTX on the expression levels of COX-2, Bcl-2 and Bax in SGC-7901 cells in vitro. To determine which genes are regulated by HM and PTX during apoptosis, the expression of COX-2, Bcl-2 and Bax were measured using western blot analysis. Exposure of gastric cancer cells to HM and PTX reduced the expression levels of COX-2 and Bcl-2, while the Bax expression levels were increased (Fig. 4). In 
addition, the combined application of HM and PTX resulted in a reduction in the COX-2 and Bcl-2 expression levels with a simultaneous increase in the Bax expression compared with the effects of either of drugs alone.

Effects of HM and PTX on the tumor growth in vivo. To further explore the effects of HM and PTX on tumor growth, a gastric xenograft tumor model was established. As shown in Fig. 5A, both HM and PTX effectively inhibited tumor growth compared to control mice. The combination groups exhibited an improved inhibition on tumor growth compared with either of the drugs used alone. In addition, the combined application of HM and PTX resulted in a statistically significant decrease in the COX-2 expression compared with the effects of either of drugs alone (Fig. 5B).

\section{Discussion}

Gastric cancer is one of the common malignant tumors in China which severely threatens human health due to its high morbidity and low early diagnosis rate (16). PTX is an important drug for treating gastric cancer, for it can effectively prolong the survival time of late gastric cancer patients and improve their living quality (17). However, a number of clinical and experimental studies in recent years have discovered that certain types of malignant tumors, including breast cancer, lung cancer, ovarian cancer and gastric cancer would primarily or secondarily resist the paclitaxe (18). It is, therefore, of great significance to explore a novel method to enhance the anticancer effect of PTX and to produce medicines which can reverse the resistance against PTX.

The present study reveals the combined administration of HM and PTX is significantly more efficient than the administration of a single drug on inhibiting proliferation inhibition and apoptosis induction. It preliminarily demonstrates that HM and PTX have synergistic effects on anticancer treatment. Therefore, in order to reach the same inhibiting effect, HM can reduce the dosage of PTX, strengthen the gastric cancer cells' sensitivity to the PTX, and weaken the toxic side effects of high-dose chemotherapeutical medicine on multiple systems of body.

A molecular target for cancer prevention and treatment that has been studied extensively in the last decade is COX-2. Our previous study demonstrated that HM significantly inhibits COX-2 expression in BGC-823 and SGC-7901 cells (14). The western blot results in the present study indicated that the combined application of HM and PTX resulted in a significant reduction in the expression of COX-2 compared with the effects of either of drugs alone in vitro and in vivo for the first time.

It is well known that tumorigenesis is due to an imbalance between cell proliferation and apoptosis (19). PCNA is a nuclear protein that is synthesized in late $\mathrm{G} 1$ and $\mathrm{S}$ phases of the cell cycle and it is used to monitor changes in cellular growth status (18). The modulation of PCNA expression is an important indicator of early changes in cellular proliferation and provides a potential mechanism by which HM and PTX may inhibit cell proliferation. Our previous study also demonstrated that treatment with NS-398, which is a selective COX-2 inhibitor, significantly reduced PCNA expression in human pancreatic carcinoma cells (20). Therefore, PTX combined with HM suppressed cell proliferation through inhibition of COX-2-associated PCNA expression in gastric cancer cells.

It has previously been shown that apoptosis is in part modulated by the Bcl-2 family (19). In the present study, it was observed that both HM and PTX down-regulated Bcl-2 expression as well as up-regulating Bax expression, while the combination of the two drugs generated an improved effect. Data from both in vivo and in vitro studies have demonstrated that up-regulation of COX-2 expression reduces the apoptosis rate by upregulating $\mathrm{Bcl}-2$ protein $(21,22)$. Our previous study demonstrated that NS-398 significantly decreases Bcl-2 protein level but increases Bax protein level in human gastric cancer cells (13). These results indicate that the combination of HM and PTX inducing apoptosis may be due to the down-regulation of COX-2 expression in gastric cancer cells.

The results of the present study indicate that the combination of HM and PTX inhibits gastric cancer development more effectively than each drug alone. PTX in combination with HM may inhibit tumor proliferation and induces apoptosis through down-regulation of cyclooxygenase-2 expression in gastric cancer. These findings may provide theoretical and experimental basis for the treatment of gastric cancer with single Chinese medicine HM and chemotherapeutic drug PTX in clinical application.

\section{References}

1. Liu SZ, Wang B, Zhang F, Chen Q, Yu L, Cheng LP, Sun XB and Duan GC: Incidence, survival and prevalence of esophageal and gastric cancer in linzhou city from 2003 to 2009. Asian Pac J Cancer Prev 14: 6031-6034, 2013.

2. Schwarz Re and Smith DD: Clinical impact of lymphadenectomy extent in resectable gastric cancer of advanced stage. Ann Surg Oncol 14: 317-328, 2007.

3. Liu W, Yang Q, Liu B and Zhu Z: Serum proteomics for gastric cancer. Clin Chim Acta 431: 179-184, 2014.

4. de Oliveira R, Deschoemaeker S, Henze AT, Debackere K, Finisguerra V, Takeda Y, Roncal C, Dettori D, Tack E, Jönsson Y, et al: Gene-targeting of Phd2 improves tumor response to chemotherapy and prevents side-toxicity. Cancer Cell 22: 263-277, 2012.

5. Wani WA, Saleem K and Haque A: Platinum compounds: A hope for future cancer chemotherapy. Anticancer Agents Med Chem 13: 296-306, 2013.

6. Sakamoto J, Matsui T and Kodera Y: Paclitaxel chemotherapy for the treatment of gastric cancer. Gastric Cancer 12: 69-78, 2009.

7. Cao MR, Li Q, Liu ZL, Liu HH, Wang W, Liao XL, Pan YL and Jiang JW: Harmine induces apoptosis in HepG2 cells via mitochondrial signaling pathway. Hepatobiliary Pancreat Dis Int 10: 599-604, 2011.

8. Liao X, Che X, Zhao W, Zhang D, Bi T and Wang G: The $\beta$-adrenoceptor antagonist, propranolol, induces human gastric cancer cell apoptosis and cell cycle arrest via inhibiting nuclear factor $\kappa B$ signaling. Oncol Rep 24: 1669-1676, 2010.

9. Abe A and Yamada H: Harmol induces apoptosis by caspase- 8 activation independently of Fas/Fas ligand interaction in human lung carcinoma H596 cells. Anticancer Drugs 20: 373-381, 2009.

10. Dai F, Chen Y, Song Y, Huang L, Zhai D, Dong Y, Lai L, Zhang T, Li D, Pang X, et al: A natural small molecule harmine inhibits angiogenesis and suppresses tumour growth through activation of $\mathrm{p} 53$ in endothelial cells. PLoS One 7: e52162, 2012.

11. Sun WH, Sun YL, Fang RN, Shao Y, Xu HC, Xue QP, Ding GX and Cheng YL: Expression of cyclooxygenase-2 and matrix metalloproteinase-9 in gastric carcinoma and its correlation with angiogenesis. Jpn J Clin Oncol 35: 707-713, 2005.

12. Chan MW, Wong CY, Cheng AS, Chan VY, Chan KK, To KF, Chan FK, Sung JJ and Leung WK: Targeted inhibition of COX-2 expression by RNA interference suppresses tumor growth and potentiates chemosensitivity to cisplatin in human gastric cancer cells. Oncol Re 18: 1557-1562, 2007. 
13. Sun WH, Zhu F, Chen GS, Su H, Luo C, Zhao QS, Zhang Y, Shao Y, Sun J and Zhou SM, et al: Blockade of cholecystokinin-2 receptor and cyclooxygenase-2 synergistically induces cell apoptosis, and inhibits the proliferation of human gastric cancer cells in vitro. Cancer Lett 263: 302-311, 2008.

14. Zhang H, Sun K, Ding J, Xu H, Zhu L, Zhang K, Li X and Sun W: Harmine induces apoptosis and inhibits tumor cell proliferation, migration and invasion through down-regulation of cyclooxygenase-2 expression in gastric cancer. Phytomedicine 15: 348-355, 2014.

15. He XP, Shao Y, Li XL, Xu W, Chen GS, Sun HH, Xu HC, Xu X, Tang D, Zheng XF, et al: Downregulation of miR-101 in gastric cancer correlates with cyclooxygenase-2 overexpression and tumor growth. FEBS J 279: 4201-4212, 2012.

16. Neugut AI, Hayek M and Howe G: Epidemiology of gastric cancer. Semin Oncol 23: 281-291, 1996.

17. Tuan TF, Tsai ML, Yeh KC, Huang HC, Chung CT, Huang CL, Han $\mathrm{CH}$, Chen $\mathrm{CP}$, Wang $\mathrm{MH}$, Shen $\mathrm{CC}$, et al: Intravenous paclitaxel against metastasis of human gastric tumors of diffuse type. Cancer Chemother Pharmacol 66: 773-783, 2010.
18. Papadaki C, Mavroudis D, Trypaki M, Koutsopoulos A Stathopoulos E, Hatzidaki D, Tsakalaki E, Georgoulias V and Souglakos J: Tumoral expression of TXR1 and TSP1 predicts overall survival of patients with lung adenocarcinoma treated with first-line docetaxel-gemcitabine regimen. Clin Cancer Res 15: 3827-3833, 2009.

19. Williams GT and Smith CA: Molecular regulation of apoptosis: Genetic controls on cell death. Cell 74: 777-779, 1993

20. Sun WH, Chen GS, Ou XL, Yang Y, Luo C, Zhang Y, Shao Y, Xu HC, Xiao B, Xue YP, et al: Inhibition of COX-2 and activation of peroxisome proliferators activated receptor $\gamma$ synergistically inhibits proliferation and induces apoptosis of human pancreatic carcinoma cells. Cancer Lett 275: 247-255, 2013.

21. Tsujii M and DuBois RN: Alterations in cellular adhesion and apoptosis in epithelial cells overexpression prostaglandin endoperoxide synthase-2. Cell 83: 493-501, 1995.

22. Sawaoka H, Tsuji S, Tsujii M, Gunawan ES, Sasaki Y, Kawano S and Hori M: Cyclooxygenase inhibitors suppress angiogenesis and reduce tumor growth in vivo. Lab Invest 79: 1469-1477, 1999. 